\title{
Draft Genome of Cissus quadrangularis to Elucidate the Medicinal Values
}

\begin{abstract}
Cissus quadrangularis is a medicinal plant belonging to the family Vitaceae and is commonly found in the tropical regions of India, Ceylon, East Africa, Malaysia and Thailand. It is referred to as Veldt Grape and it is a traditional medicine for joint and bone health and shows promise in promoting bone growth rates. The whole plant is used in oral re-hydration, while the leaf, stem, and root extracts of this plant are important in the management of various ailments. This perennial plant has been shown to be very effective in various human diseases such as osteoporosis, asthma, cough, hemorrhoids and gonorrhea. Here we report the first draft genome sequence of Cissus quadrangularis of cultivar variant I - TUH No. 189 which will provide the genetic basis for studying the important active components of the plant. We have assembled a draft genome of $313 \mathrm{Mb}$ which covers nearly $64 \%$ of the nearest genome of Vitis vinifera and 36,325 proteins were identified through genome annotation.
\end{abstract}

Keywords: Secondary metabolites; Genome annotation; Repeat elements; Assembly

Received: January 01, 2016; Accepted: January 22, 2016; Published: Janaury 25, 2016

\section{Introduction}

Next-generation sequencing (NGS) technologies have permitted the rapid and efficient development of genomic resources for non-model or orphan plant species [1-3]. The investigation of indigenous plant genomes to decipher the genomic characterization and the genetic basis of its active compounds with proven medicinal efficacy is a relatively new area of exploration. Plant secondary metabolites from a large repertoire of plants are implicated in a wide range of human diseases, including cancer, diabetes, bacterial, parasitic and fungal infections. The pathways involved in the production of the metabolites need to be deciphered at a molecular level using genome information. Whole genome analysis of important plant species, native to India, is the first big step in achieving a complete molecular understanding of their medicinal value. The genomic information is very limited in the area of medicinal plants and provides us with a golden opportunity to investigate the genetic makeup and embark on a high-throughput whole genome analysis.

Cissus quadrangularis, belonging to the family Vitaceae, is commonly found in tropical regions of India, Ceylon, East Africa, Malaysia and Thailand [4]. It is an annual or perennial herb, entire leaves, buff colored with greenish ting and requires warm tropical climate and propagated by stem cuttings. It is commonly
Gandhimathi A', Margaret Sunitha S, Pritha Ghosh ${ }^{1}$, Harini K', Subramanian Hariprasanna P', lyer Meenakshi S', Adwait Joshi G1,3, Karpe Snehal D', Jarjapu Mahita', Manoharan Malini', Oommen Mathew $\mathrm{K}^{1,2}$, Eshita Mutt', Mahantesha Naika', Nithin Ravooru', Sathyanarayanan Nitish', Shaik Naseer Pasha ${ }^{1,3}$, Upadhyayula Raghavender S', Rajas Rao M', Mohamed Shafi K', Prashant Shingate N ${ }^{1}$, Anshul Sukhwal ${ }^{1,2}$, Atul Upadhyay K', Rithvik Vinekar $\mathbf{S}^{1}$ and

\section{Ramanathan Sowdhamini ${ }^{1}$}

1 National Centre for Biological Sciences (TIFR), GKVK Campus, Bangalore, Karnataka, India

2 SASTRA University, Tirumalaisamudram, Thanjavur, Tamil Nadu, India

3 Manipal University, Madhav Nagar, Manipal, Karnataka 576104, India

Corresponding author:

Ramanathan Sowdhamini

$\equiv$ mini@ncbs.res.in

National Centre for Biological Sciences (TIFR), GKVK Campus, Bangalore-560 065, Karnataka, India.

Citation: Gandhimathi A, Sunitha SM, Ghosh P, et al. Draft Genome of Cissus quadrangularis to Elucidate the Medicinal Values. Herb Med. 2016, 2:1. 
referred to as the "bone setter," "Asthisamdhani" in Sanskrit and "Hadjod" in Hindi. It has been used by common folk in India for promoting the fracture healing process [5]. All parts of plants are used for medicinal purpose in a form of extracts and powders [6]. Traditionally, Cissus quadrangularis have been used for many years to promote bone and tissues healing, as an analgesic, to treat infections, as an anabolic, and to promote weight loss and weight management [7]. In addition to bone healing property, Cissus quadrangularis has been shown to have antiosteoporotic [8], anti-oxidant [9], anti-microbial [10], analgesic and antiinflammatory [11], insulin-resistant [12], anti-ulcer and gastro protective properties [13].

Phytochemical analysis of Cissus quadrangularis revealed the presence of several medicinally important plant secondary metabolites including $\alpha$ - and $\beta$-amyrin, $\beta$-sitosterol, Quercetin, kaempferol, stilbene derivatives, quadrangularins $A, B, C$, resveratrol and large amounts of Calcium and Vitamin-C [14-16]. All the literature discuss about the information of particular plant tissues and specific secondary metabolites for their implicated role in the cure of diseases. Till now, there has been no clear information of the presence of secondary metabolites at the genetic level and at the level of metabolic pathways secondary metabolites and the metabolic pathways at the genetic level for Cissus quadrangularis. Here, we report the draft genome of Cissus quadrangularis and these data will facilitate more comprehensive analysis to decipher genes and pathways involved in the production of medicinally important secondary metabolites.

\section{Methods}

The genomic DNA of Cissus quadrangularis was extracted from the leaf samples using Qiagen Plant genomic DNA extraction kit. The final DNA sample was eluted with nuclease free water and the quality of the samples were tested using Nanodrop (GE NanoVue). The concentration of the extracted samples was $\sim 50$ $\mathrm{ng} / \mu \mathrm{L}$ as measured by the Qubit Fluorometer. The draft genome was sequenced using paired end (PE) library with an insert size of $350 \mathrm{bp}$ by Illumina HiSeq 1000 platform. We obtained 336,794 reads from the PE library and the FASTX-Toolkit (http://hannonlab. cshl.edu/fastx toolkit/) was used for the quality check of reads. Low quality reads were trimmed and quality score below Q20 were rejected. The good quality reads were further used for de novo genome assembly using SOAP denovo2 [17]. Different K-mers from 23 to 59, with a step size of two, were applied for the genome assembly. The assembled draft genome was further utilized for functional annotation using the MAKER program [18] with AUGUSTUS gene prediction tool [19] which was trained on Arabidopsis thaliana.

\section{Results}

We obtained the best assembly of $312 \mathrm{Mb}$ at a K-mer of 31. The scaffold N50 is $5.7 \mathrm{~Kb}, 6206$ scaffolds which are more than 100 $\mathrm{bp}$ in length and with the longest scaffold length of $111 \mathrm{~Kb}$ (Table 1). Repeat elements in the assembled genome were identified using Repeat Scout (version 1.0.5) [20] and Repeat-Masker (http://www.repeatmasker.org/) 18.20\% (version 4.0.3). of the assembled genome was masked due to the existence of repeat elements.

A total of 62,719 genes were initially predicted through the MAKER pipeline. After redundancy removal at $100 \%$ sequence identity using CD-HIT, 36,325 proteins were recognized as the final proteome. The CEGMA program [21] was implemented to identify the core set of essential eukaryotic genes. Around 53\% (132 out of 248) of these essential genes were identified from our genome assembly. The draft genome of Cissus quadrangularis is deposited at NCBI (Bio sample accession number SAMN03223842). Further works, on the characterization of metabolite pathway-related genes, to elucidate their functional context and the orthology relationship of the predicted proteins, are in progress. 


\section{References}

1 Goodstein DM, Shu S, Howson R, Neupane R, Hayes RD, et al. (2012) Phytozome: a comparative platform for green. Plant genomics. Nucleic Acids Res 40: D1178-1186.

2 Hamilton JP, Buell CR (2012) Advances in plant genome sequencing. Plant J Cell Mol Biol 70: 177-190.

3 Imelfort M, Edwards D (2009) De novo sequencing of plant genomes using second-generation technologies. Brief Bioinform 10: 609-618.

4 Khan SS, Chaghtai SA (1991) Ethnomedicobotany of Cissus quadrangularis Linn. Orient J Chem 7: 170-172.

5 Prasad GC, Udupa KN (1963) Effect of cissus quadrangularis on the healing of cortisone treated fractures. Indian J Med Res 51: 667-676.

6 Stohs SJ, Ray SD (2013) A Review and Evaluation of the Efficacy and Safety of Cissus quadrangularis Extracts. Phytother Res 27: 1107-1114.

7 Mishra G, Srivastava S, Nagori BP (2010) Pharmacological and therapeutic activity of Cissus quadrangularis: An overview. Int J PharmTech Res 2: 1298-1310.

8 Shirwaikar A, Khan S, Malini S (2003) Antiosteoporotic effect of ethanol extract of Cissus quadrangularis Linn. on ovariectomized rat. J Ethnopharmacol 89: 245-250.

9 Jainu M, Devi CSS (2005) In Vitro and In Vivo Evaluation of FreeRadical Scavenging Potential of Cissus quadrangularis. Pharm Biol 43: 773-779.

10 Chidambara Murthy KN, Vanitha A, Mahadeva Swamy $M$, Ravishankar GA (2003) Antioxidant and antimicrobial activity of Cissus quadrangularis L. J Med Food 6: 99-105.

11 Panthong A, Supraditaporn W, Kanjanapothi D, Taesotikul T, Reutraku V (2007) Analgesic, anti-inflammatory and venotonic effects of Cissus quadrangularis Linn. J Ethnopharmacol 110: 264-270.
12 Chidambaram J, Carani Venkatraman A (2010) Cissus quadrangularis stem alleviates insulin resistance, oxidative injury and fatty liver disease in rats fed high fat plus fructose diet. Food Chem Toxicol 48 : 2021-2029.

13 Jainu M, Devi CSS (2006) Gastroprotective action of Cissus quadrangularis extract against NSAID induced gastric ulcer: Role of proinflammatory cytokines and oxidative damage. Chem Biol Interact 161: 262-270.

14 Enechi OC, Odonwodo I (2004) An Assessment of the Phytochemical and Nutrient Composition of the Pulverized Root of Cissus Quadrangularis. Bio-Res 1: 63-68.

15 Mehta M, Kaur N, Bhutani KK (2001) Determination of marker constituents from Cissus quadrangularis Linn. and their quantitation by HPTLC and HPLC. Phytochem Anal PCA 12: 91-95.

16 Sen M, Dash B (2012) A review on phytochemical and pharmacological aspects of Cissus quadrangularis L. Int J Green Pharm 6: 169.

17 Xie Y, Wu G, Tang J, Luo R, Patterson J, et al. (2014) SOAP denovoTrans: de novo transcriptome assembly with short RNA-Seq reads. Bioinforma Oxf Engl 30: 1660-1666.

18 Cantarel BL, Korf I, Robb SMC, Parra G, Ross E, et al. (2008) MAKER: an easy-to-use annotation pipeline designed for emerging model organism genomes. Genome Res 18: 188-196.

19 Stanke M, Morgenstern B (2005) AUGUSTUS: a web server for gene prediction in eukaryotes that allows user-defined constraints. Nucleic Acids Res 33: W465-467.

20 Price AL, Jones NC, Pevzner PA (2005) De novo identification of repeat families in large genomes. Bioinforma Oxf Engl 21 Suppl 1: i351-358.

21 Parra G, Bradnam K, Korf I (2007) CEGMA: A pipeline to accurately annotate core genes in eukaryotic genomes. Bioinforma Oxf Engl 23: 1061-1067. 